\title{
Fungos liquenizados da Mata Atlântica, no sul do Brasil
}

Suzana Maria de Azevedo Martinss ${ }^{1,3}$, Márcia Isabel Käffer ${ }^{1}$, Camila Ribeiro Alves² e Viviane Camejo Pereira²

Recebido em 10/12/2008. Aceito em 11/03/2011

\begin{abstract}
RESUMO
(Fungos liquenizados no bioma Mata Atlântica, no sul do Brasil). São apresentados resultados de levantamento baseado em dados de coletas de fungos liquenizados em algumas localidades de Mata Atlântica na região sul do Brasil. Foram identificados 88 táxons distribuídos em 18 famílias e 36 gêneros; destes, dois são citações novas para o Brasil e um é nova ocorrência para o Rio Grande do Sul.
\end{abstract}

Palavras-chave: Composição de espécies, liquens, Mata Atlântica, Rio Grande do Sul

\begin{abstract}
(Lichenized fungi in the Atlantic Rainforest biome, in Southern Brazil). The results of a survey based on data of lichenized fungi collections from some localities in the Atlantic Rainforest of South Brazil are presented. A total of 88 taxa distributed in 18 families and 36 genera were identified; from these, two are new records for Brazil and one is a new occurrence for the State of Rio Grande do Sul.
\end{abstract}

Key words: Species composition, lichen, Mata Atlântica, Rio Grande do Sul

\section{Introdução}

Nas últimas décadas, o desenvolvimento acelerado de muitos países vem ocasionando problemas sérios para os ecossistemas. Em vista dessa problemática, a conservação da biodiversidade de muitos biomas tem sido enfocada. No Brasil, um dos biomas mais afetados é a Mata Atlântica que atualmente corresponde a $8 \%$ de sua extensão original, caracterizada pela alta fragmentação de habitats e pela biodiversidade (IBGE 2008).

A Mata Atlantica ocupa toda a faixa continental atlântica leste brasileira e se estende para o interior no Sudeste e Sul do País, correspondendo a uma área total de 13,04\%. Nesta última região predominam as florestas estacionais semideciduais, e as florestas ombrófilas densas e mistas (com araucária). Em ambos os conjuntos florestais ocorrem, em menor proporção, as florestas estacionais deciduais (IBGE 2008).

As constantes ações antrópicas nestas áreas florestais ocasionam perturbações nos ecossistemas, atingindo diversas comunidades, entre elas, a micota liquenizada. Mesmo que algumas comunidades sejam capazes de sobreviver a este tipo de impacto, muitas populações podem sofrer extinção local de espécies que são restritas aos ecossistemas com baixos regimes de perturbações (Will-Wolf et al. 2002). Outras podem sofrer perda de diversidade e se encontrarem dominadas por espécies mais resistentes a ambientes perturbados (Jüriado et al. 2003, Brunialti \& Giordani 2003). A composição e diversidade das espécies liquênicas pode ser determinada pelas características do substrato (Brodo 1973; Marcelli 1996; Schmidt et al. 2001), disponibilidade de forófitos (Lesica et al. 1991), constituição dos macro e micro-nutrientes (Hawksworth 1975), além dos fatores climáticos (Brunialti \& Giordani 2003).

Para as áreas de Mata Atlântica no Rio Grande do Sul há trabalhos sobre fungos liquenizados realizados por Osorio (1982), Osorio \& Fleig (1984; 1986a; 1986b), Fleig (1985, 1990, 1999), Fleig \& Grüninger (2000), Käffer \& MartinsMazzitelli (2005) e Fleig \& Grüninger (2008).

O estudo da composição da micota liquenizada faz parte de subprojeto de avaliação de áreas prioritárias para a conservação da biodiversidade em nível de bioma, entre os quais a Mata Atlântica (Ministério do Meio Ambiente 2002). Este trabalho tem por objetivo apresentar informações sobre a micota liquenizada ocorrente na área de abrangência do bioma Mata Atlântica na região sul do País.

\footnotetext{
Fundação Zoobotânica do Rio Grande do Sul, Museu de Ciências Naturais, Porto Alegre, RS, Brasil

2 Universidade Luterana do Brasil, Curso de Ciências Biológicas, Canoas, RS, Brasil

3 Autor para correspondência: suzana.martins@fzb.rs.gov.br
} 


\section{Material e métodos}

Para o desenvolvimento do trabalho foram analisados os dados de coleta dos anos: 1993, 1994, 1999, 2000, 2002, 2005, 2006 e 2007, nos municípios de Cambará do Sul, Jaquirana, Maquiné e São Francisco de Paula, Rio Grande do Sul. Foram realizadas coletas anuais através do método do caminhamento (Filgueiras et al. 1994) ao longo das trilhas, encostas rochosas, interiores e bordas da mata onde foram coletadas amostras de material corticícola, terrícola e saxícola.

As amostras táxons foram analisadas com o auxílio de microscópio estereoscópico e de luz; foram feitos cortes no talo e frutificações, além de testes de coloração empregados em taxonomia de fungos liquenizados. Os táxons foram identificados com base principalmente nas seguintes referências: Ahti 2000; Aptroot 1987; Arvidsson 1982; Awasthi 1988; Dodge 1964; Eliasaro 1992; Eliasaro \& Adler 2000; Elix 1994; Fleig 1997; 1999; Fleig et al. 1995; Galloway 1985a; 1985b; 1986a; 1986b; 1988; 1994; 1997; 1998a; 1998b; 2001; Galloway \& James 1980; 1986; Jorgensen 1978; Jorgensen \& James 1983; 2001; Krog 1982; McDonald et al. 2003; Moberg 1990; Ribeiro 1998; Scutari 1992; 1995a; 1995b; Sérusiaux 1983; Sierk 1964; Sipman 2002; Swinscow \& Krog 1998; Verdon et al. 1992; Wolseley 1991; Yoshimura \& Osorio 1975, além de outros trabalhos taxonômicos citados em Marcelli et al. (1998).

O material coletado encontra-se no Herbário Prof. Dr. R. H. Alarich Schultz (HAS) do Museu de Ciências Naturais da Fundação Zoobotânica do Rio Grande do Sul, com os seguintes números de registro: 43.393 a 43.441, 43.994 a $44.164,44.796$ a $44.803,46.050$ a $46.183,46.259$ a 46.265 , 46.471 a 46.478.

\section{Resultados e discussão}

Nas áreas amostradas foram registrados 88 táxons de fungos liquenizados distribuídos em 18 famílias e 36 gêneros; destes Pannaria aff. ignobilis e Peltigera polydactyla são citações novas para o Brasil e Coenogonium cf. moniliforme é nova ocorrência para o Rio Grande do Sul (Tab. 1).

Pannaria aff. ignobilis possui ampla distribuição na Europa, Norte da África e Estados Unidos (GBIF 2009). Caracteriza-se pelo hábito corticícola, ocorrendo provavelmente em regiões com cerca de 1000 metros de altitude (Swinscow \& Krog 1998). No presente trabalho a espécie foi encontrada sobre o tronco de árvores de ambiente úmido e sombreado entre altitudes que variaram de 850 a 1100 metros.

Peltigera polydactyla é espécie cosmopolita, amplamente distribuída no Hemisfério Norte ocorrendo em solos, musgos, rochas e também na base dos troncos de árvores de florestas úmidas (Thomson Jr. 1950). Holtan-Hartwing (1993) relata que muitas vezes esta espécie é encontrada crescendo sobre rochas cobertas de musgos localizadas em cabeceiras de rios e em troncos situados em matas ciliares atingidos por períodos de inundação. Na Mata Atlântica foi coletada sobre rochas e barrancos úmidos de áreas abertas.

Cáceres (2007) cita Coenogonium cf. moniliforme para área de Caatinga, em Sergipe. De acordo com Xavier Filho et al. (1983) esta espécie encontra-se distribuída nas regiões Norte, Nordeste, Sudeste e Sul do Brasil, sendo normalmente encontrada em matas tropicais e subtropicais. No bioma amostrado foi encontrado nos troncos de árvores de ambiente úmido e sombreado.

Nas áreas abertas e nas bordas de mata, onde ocorre mais iluminação, foi possível notar maior ocorrência de espécies, incluídas as do gênero Parmotrema (Parmeliaceae), que se destacou por apresentar o maior número de táxons (35) correspondendo a 39,7\% do total, seguida de Lobariaceae com 17 táxons (19,3\%) e Cladoniaceae com 16 táxons (18,2\%).

A maior ocorrência de Parmeliaceae pode estar associada ao fato da família apresentar um grande número de táxons, pois se estima aproximadamente a existência de 2400 espécies distribuídas em 85 gêneros (Louwhoff \& Elix 1999; Blanco et al. 2005). Além disso, Parmeliaceae possui maior representatividade devido aos vários estudos registrados sobre esta família no Estado, talvez pela sua morfologia que se destaca diante de táxons menos conhecidos como, os crostosos, fazendo com que exista um grande número de amostras disponíveis nos herbários, o que facilita a identificação. A maior representatividade desta família também foi reportada em outros trabalhos (Fleig 1990; Martins - Mazzitelli et al. 1999; Fleig \& Grüninger 2000; Käffer \& Martins-Mazzitelli 2005). Marcelli et al. (1998) citam que em florestas de Mata Atlântica, os ramos e galhos expostos à luz e os troncos nas bordas das florestas apresentam uma grande diversidade de Parmeliaceae (Parmotrema, Rimelia, Hypotrachyna, Punctelia e Parmelinopsis), Collemataceae (Leptogium), Physciaceae (Heterodermia), Lobariaceae e Graphidaceae, semelhante ao encontrado neste trabalho.

O gênero Sticta se destacou por apresentar o maior número de espécies (10), Parmotrema ficou em segunda posição com nove, Cladonia com sete e Rimelia com cinco. Do total de espécimes coletados $68,2 \%$ são foliosos, seguido dos fruticosos (10,2\%), dimórficos (10,2\%), crostosos $(7,9 \%)$, esquamulosos (2,3\%) e filamentosos (1,2\%).

Nas áreas de mata úmida e sombreada foram encontrados $23,9 \%$ do total de espécimes de fungos liquenizados, especialmente dos gêneros Leptogium, Sticta, Collema e Pannaria, que são característicos deste tipo de ambiente (Arvidsson 1982; Sipman \& Harris 1989; Wolseley 1991).

A composição de espécies liquênicas para o bioma Mata Atlântica, no sul do Brasil, ainda carece de estudos. Investigações na mesma região relatam 412 espécies (Spielmann 2006), enquanto para Marcelli (1998) seriam esperadas, para a área de abrangência de todo o bioma, 2100 espécies.

A Mata Atlântica apresenta um clima extremamente úmido devido à alta e constante precipitação de água, o que torna o ambiente desfavorável ao desenvolvimento de 
Tabela 1. Fungos liquenizados no bioma Mata Atlântica, no sul do Brasil. 1- Mata úmida na sombra, 2 - Em clareiras e florestas abertas, local seco e ensolarado, 3 - Em ramos finos na beira da mata, ensolarado, 4 - Em barrancos ou rochas, local úmido com luz, ensolarado, 5 - No solo na beira da mata, 6 - Em troncos ou rochas na beira da mata, ensolarado, 7 - Na mata, local pouco sombreado. Legenda: • Novo registro RS, $\bullet$ Citação nova Brasil; Fol. = folioso; Dim = dimórfico; Frut. $=$ fruticoso; Fil. $=$ filamentoso; Ter. $=$ terrícola; Sax.= saxícola; Cort. $=$ corticícola.

\begin{tabular}{|c|c|c|c|c|c|c|c|c|c|c|}
\hline \multirow{2}{*}{ Família } & \multirow{2}{*}{ Táxons } & \multicolumn{7}{|c|}{ Locais de coleta } & \multirow{2}{*}{ Hábitat } & \multirow{2}{*}{ Hábito } \\
\hline & & 1 & 2 & 3 & 4 & 5 & 6 & 7 & & \\
\hline ARTHONIACEAE & Cryptothecia rubrocincta (Ehrenb.) G. Thor & $\mathrm{x}$ & & & & & & & Cort. & Crost. \\
\hline \multirow[t]{2}{*}{ BIATORACEAE } & Phyllopsora chlorophaea (Müll. Arg.) Zahlbr. & $\mathrm{x}$ & & & & & & & Cort. & Esq. \\
\hline & Phyllopsora cf. parvifolia (Pers.) Müll.Arg .var. breviuscula (Nyl.) Brako & $\mathrm{x}$ & & & & & & & Cort. & Esq. \\
\hline BRIGANTIAECEAE & Brigantiaea leucoxantha (Spreng.) R. Sant. \& Haffelner & & $\mathrm{x}$ & & & & & & Cort. & Crost. \\
\hline \multirow[t]{9}{*}{ CLADONIACEAE } & Cladia aggregata (Sw.) Nyl. & & & & & $\mathrm{x}$ & & & Ter. & Dim. \\
\hline & Cladina confusa (R. Sant.) Follmann \& Ahti & & & & & $\mathrm{x}$ & & & Ter. & Dim. \\
\hline & Cladonia ceratophylla (Sw.) Spreng. & & & & & & $\mathrm{x}$ & & Cort. & Dim. \\
\hline & Cladonia macilenta Hoffm. & & & & & $\mathrm{x}$ & & & Ter. & Dim. \\
\hline & Cladonia aff. ochracea Scriba & & & & $\mathrm{x}$ & & & & Saxíc./Ter. & Dim. \\
\hline & Cladonia rappii A. Evans & & & & & $\mathrm{x}$ & & & Ter. & Dim. \\
\hline & Cladonia solida Vain. & & & & & & $\mathrm{x}$ & & Saxíc. & Dim. \\
\hline & Cladonia cf. subradiata (Vain.) Sandst. & & $\mathrm{x}$ & & & & & & Cort./Ter. & Dim. \\
\hline & Cladonia subsquamosa Kremp. & & & & & & $\mathrm{x}$ & & Cort. & Dim. \\
\hline \multirow[t]{2}{*}{ COCCOCARPIACEAE } & Coccocarpia erythroxyli (Spreng.) Swinscow \& Krog & & $\mathrm{x}$ & & & & & & Cort. & Fol. \\
\hline & Coccocarpia pellita (Ach.) Müll.Arg. ex R. Sant. & & $\mathrm{x}$ & & & & & & Cort. & Fol. \\
\hline \multirow[t]{2}{*}{ COLLEMATACEAE } & Leptogium azurem (Sw.) Mont. & $\mathrm{x}$ & & & & & & & Cort. & Fol. \\
\hline & Leptogium chloromelum (Sw.) Nyl. & $\mathrm{x}$ & & & & & & & Cort. & Fol. \\
\hline GRAPHIDACEAE & Sarcographa sp. & & & $\mathrm{x}$ & & & & & Cort. & Crost. \\
\hline GYALECTACEAE & Coenogonium cf. moniliforme Tuck.* & $\mathrm{x}$ & & & & & & & Cort. & Fil. \\
\hline \multirow[t]{17}{*}{ LOBARIACEAE } & Lobaria discolor (Bory ex Delise) Hue & & & & & & $\mathrm{x}$ & & Cort./Saxíc. & Fol. \\
\hline & Lobaria erosa (Eschw.) Nyl. & & & & & & $\mathrm{x}$ & & Cort. & Fol. \\
\hline & Lobaria intermedia (Nyl.)Vain. & & & & & & $\mathrm{x}$ & & Cort. & Fol. \\
\hline & Lobaria tenuis Vain. & & & & & & $\mathrm{x}$ & & Cort. & Fol. \\
\hline & Pseudocyphellaria aurata (Ach.) Vain. & $\mathrm{x}$ & & & & & $\mathrm{x}$ & & Cort. & Fol. \\
\hline & Pseudocyphellaria cf. berteriana (G. Forster) D.J. Galloway \& P. James & $\mathrm{x}$ & & & & & & & Cort. & Fol. \\
\hline & Pseudocyphellaria clathrata (De Not.) Malme & & & & & & $\mathrm{x}$ & & Cort. & Fol. \\
\hline & Sticta ambavillaria (Bory) Ach. & & & & & & & $\mathrm{x}$ & Cort. & Fol. \\
\hline & Sticta damaecornis (Sw.) Ach. & & & & & & & $\mathrm{x}$ & Cort. & Fol. \\
\hline & Sticta fuliginosa(Dicks.) Ach. & & & & & & $\mathrm{x}$ & & Cort./Saxíc. & Fol. \\
\hline & Sticta sinuosa Pers. & & & & & & & $\mathrm{x}$ & Cort. & Fol. \\
\hline & Sticta variabilis (Bory) Ach. & & & & & & & $\mathrm{x}$ & Cort. & Fol. \\
\hline & Sticta sp. 1 & $\mathrm{x}$ & & & & & & & Cort. & Fol. \\
\hline & Sticta sp. 2 & $\mathrm{x}$ & & & & & & & Cort. & Fol. \\
\hline & Sticta sp. 3 & & & & & & & & Cort. & Fol. \\
\hline & Sticta sp. 4 & & & & & & $\mathrm{x}$ & & Cort. & Fol. \\
\hline & Sticta sp. 5 & $\mathrm{x}$ & & & & & & & Cort. & Fol. \\
\hline
\end{tabular}


Tabela 1. Continuação

\begin{tabular}{|c|c|c|c|c|c|c|c|c|c|c|}
\hline \multirow{2}{*}{ Família } & \multirow{2}{*}{ Táxons } & \multicolumn{7}{|c|}{ Locais de coleta } & \multirow{2}{*}{ Hábitat } & \multirow{2}{*}{ Hábitc } \\
\hline & & 1 & 2 & 3 & 4 & 5 & 6 & 7 & & \\
\hline \multirow[t]{4}{*}{ PANNARIACEAE } & Collema fasciculare (L.) Wigg. & $\mathrm{x}$ & & & & & & & Cort. & Fol. \\
\hline & Pannaria aff. ignobilis Anzi ${ }^{* *}$ & $\mathrm{x}$ & & & & & & & Cort. & Fol. \\
\hline & Pannaria rubiginosa (Ach.) Bory & $\mathrm{x}$ & & & & & & & Cort. & Fol. \\
\hline & Pannaria sp. 1 & $\mathrm{x}$ & & & & & & & Cort. & Fol. \\
\hline \multirow[t]{35}{*}{ PARMELIACEAE } & Bulbothrix ventricosa (Hale \& Kurok.) Hale ** & & & $\mathrm{x}$ & & & & & Cort. & Fol. \\
\hline & Canomaculina muelleri (Vain.) Elix \& Hale & & & & & & $\mathrm{x}$ & & Cort./Saxíc. & Fol. \\
\hline & Canomaculina pilosa (Stizenb.) Elix \& Hale & & & & & & $\mathrm{x}$ & & Cort. & Fol. \\
\hline & Canoparmelia texana (Tuck.) Elix \& Hale & & $\mathrm{x}$ & & & & & & Cort. & Fol. \\
\hline & Everniastrum sp. & & & & & $\mathrm{x}$ & & & Cort. & Frut. \\
\hline & Hypotrachyna dactylifera (Vain.) Hale & & $\mathrm{x}$ & & & & & & Cort. & Fol. \\
\hline & Hypotrachyna imbricatula (Zahlbr.) Hale & & & & & & $\mathrm{x}$ & & Cort. & Fol. \\
\hline & Hypotrachyna peruviana (Nyl.) Hale & & & & & & $\mathrm{x}$ & & Cort. & Fol. \\
\hline & Hypotrachyna sp. 2 & & & & & & $\mathrm{x}$ & & Cort. & Fol. \\
\hline & Megalospora sp. & & $\mathrm{x}$ & & & & & & Cort. & Crost. \\
\hline & Parmelinopsis horrescens (Taylor) Elix \& Hale & & & & & & $\mathrm{x}$ & & Cort. & Fol. \\
\hline & Parmotrema aff. argentinum (Kremp.) Hale & & $\mathrm{x}$ & & & & & & Cort. & Fol. \\
\hline & Parmotrema aff. sorediomaculiferum Marcelli \& Canêz & & $\mathrm{x}$ & & & & & & Cort. & Fol. \\
\hline & Parmotrema catarinae Hale & & & & & & $\mathrm{x}$ & & Cort. & Fol. \\
\hline & Parmotrema cetratum (Ach.) Hale & & $\mathrm{x}$ & & & & & & Cort. & Fol. \\
\hline & Parmotrema chinense (Osbeck) Hale \& Ahti & & & & & & $\mathrm{x}$ & & Cort. & Fol. \\
\hline & Parmotrema eciliatum (Nyl.) Hale & & & $\mathrm{x}$ & & & & & Cort. & Fol. \\
\hline & Parmotrema homotomum (Nyl.) Hale & & $\mathrm{x}$ & & & & & & Cort. & Fol. \\
\hline & Parmotrema macrocarpum(Pers.) Hale & & $\mathrm{x}$ & & & & & & Cort. & Fol. \\
\hline & Parmotrema mantiqueirense Hale & & & & & & $\mathrm{x}$ & & Cort./Saxíc. & Fol. \\
\hline & Parmotema melanothrix (Mont.) Hale & & $\mathrm{x}$ & $\mathrm{x}$ & & & & & Cort. & Fol. \\
\hline & Parmotrema mellissii (Dodge) Hale & & $\mathrm{x}$ & & & & & & Cort./Saxíc. & Fol. \\
\hline & Parmotrema praesorediosum (Nyl.) Hale & & $\mathrm{x}$ & $\mathrm{x}$ & & & & & Cort. & Fol. \\
\hline & Parmotrema reticulatum (Taylor) Hale \& A. Fletcher & & $\mathrm{x}$ & & & & & & Cort. & Fol. \\
\hline & Parmotrema simulans (Hale) Hale & & $\mathrm{x}$ & & & & & & Cort. & Fol. \\
\hline & Punctelia cf. reddenda (Stirt.) Krog & & $\mathrm{x}$ & & & & & & Cort. & Fol. \\
\hline & Punctelia graminicola (B. de Lesd.) Egan & & $\mathrm{x}$ & & & & & & Cort. & Fol. \\
\hline & Relicina abstrusa (Vain.) Hale & & & & & & $\mathrm{x}$ & & Cort. & Fol. \\
\hline & Usnea sp. 1 & & $\mathrm{x}$ & & & & $\mathrm{x}$ & & Cort. & Frut. \\
\hline & Usnea sp. 2 & & $\mathrm{x}$ & & & & $\mathrm{x}$ & & Cort. & Frut. \\
\hline & Usnea sp. 3 & & $\mathrm{x}$ & & & & $\mathrm{x}$ & & Cort. & Frut. \\
\hline & Usnea sp. 4 & & $\mathrm{x}$ & & & & $\mathrm{x}$ & & Cort. & Frut. \\
\hline & Xanthoparmelia cf. conglomerata Canêz \& Marcelli & & & & & & $\mathrm{x}$ & & Cort./Saxíc. & Fol. \\
\hline & Xanthoparmelia hypopsila (Mull.Arg.) Hale & $\mathrm{x}$ & & & & & & & Saxíc. & Fol. \\
\hline & Xanthoparmelia subramigera (Gyeln.) Hale & & & & $\mathrm{x}$ & & & & Saxíc. & Fol. \\
\hline PELTIGERACEAE & Peltigera polydactyla (Neck.) Hoffm. & & & & $\mathrm{x}$ & & & & Saxíc./Ter. & Fol. \\
\hline
\end{tabular}


Tabela 1. Continuação

\begin{tabular}{|c|c|c|c|c|c|c|c|c|c|}
\hline \multirow{2}{*}{ Família } & \multirow{2}{*}{ Táxons } & \multicolumn{6}{|c|}{ Locais de coleta } & \multirow{2}{*}{ Hábitat } & \multirow{2}{*}{ Hábito } \\
\hline & & 1 & 2 & 3 & 45 & 6 & 7 & & \\
\hline \multirow[t]{4}{*}{ PHYSCIACEAE } & Buellia sp. & $\mathrm{x}$ & & & & & & Cort. & Crost. \\
\hline & Heterodermia diademata (Taylor) Awasthi & $\mathrm{x}$ & & & & & & Cort. & Fol. \\
\hline & Heterodermia obscurata (Nyl.) Trevis. & & & & & $\mathrm{x}$ & & Cort. & Fol. \\
\hline & Heterodermia vulgaris (Vain.) Follmann \& Redón & & & & & $\mathrm{x}$ & & Cort. & Fol. \\
\hline \multirow[t]{2}{*}{ RAMALINACEAE } & Ramalina celastri (Spreng.) Krog \& Swinscow & & $\mathrm{x}$ & & & & & Cort. & Frut. \\
\hline & Ramalina prolifera Taylor & & $\mathrm{x}$ & & & & & Cort. & Frut. \\
\hline \multirow[t]{2}{*}{ TELOSCHISTACEAE } & Teloschistes cf. cymbalifer (Eschw.) Müll.Arg. & & $\mathrm{x}$ & & & & & Cort. & Frut. \\
\hline & Teloschistes exilis (Michx.) Vain. & & $\mathrm{x}$ & & & & & Cort. & Frut. \\
\hline THELEPHORACEAE & Dictyonema glabratum (Spreng.) D. Hawksw. & & $\mathrm{x}$ & & & & & Saxíc./Ter. & Fol. \\
\hline TRYPETHELIACEAE & Trypethelium sp. & $\mathrm{x}$ & & & & & & Cort. & Crost. \\
\hline VERRUCARIACEAE & Verrucaria sp. & $\mathrm{x}$ & & & & & & Cort. & Crost. \\
\hline
\end{tabular}

muitas espécies liquênicas; contudo, nas bordas da mata e clareiras, locais onde ocorre alta incidência luminosa, uma grande quantidade de liquens cobre os substratos competindo com as briófitas (Marcelli 1998).

Considerando os resultados do levantamento realizado, é possível caracterizar os locais de estudo como ambientes favoráveis para a formação e desenvolvimento de fungos liquenizados, sendo a ocorrência de áreas mais abertas e consequentemente mais iluminadas, um fator muito importante que influencia na composição de espécies de macroliquens.

Os fatores ambientais influenciam diretamente a distribuição de liquens ao longo e ao redor dos troncos, principalmente a luz e a umidade (Marcelli 1987). Além disso, as características físico-químicas da casca das árvores, tais como, textura, dureza, retenção de água, $\mathrm{pH}$ e composição de macro e micro nutrientes são fundamentais para o estabelecimento das comunidades liquênicas (Brodo, 1973; Marcelli, 1996; Nash III, 1997; Martins, 2006). Luz direta, temperatura moderada ou fria, umidade constante (com períodos de menos umidade) e uma atmosfera pura e limpa, são os fatores climáticos mais favoráveis para o desenvolvimento de liquens (Hawksworth, 1975). Portanto, qualquer interferência humana que venha a provocar alterações neste ambiente florestal acaba por criar novos habitats para as espécies raras ou para as oportunistas modificando a estrutura da comunidade liquênica.

Para que maior diversidade de fungos liquenizados seja preservada, é de suma importância a conservação destas formações florestais tão ameaçadas pelas ações antrópicas.

\section{Agradecimentos}

Ao colega Cleodir Mansan pelo auxílio no trabalho de campo e à acadêmica em biologia Natália Koch pela elaboração do abstract.

\section{Referências bibliográficas}

Ahti, T. 2000. Cladoniaceae. Flora Neotropica Monograph 78. New York. New York Botanical Garden, Bronx.

Aptroot, A. 1987. Flora of the Guianas. Koenigstein, Koeltz Scientific Books.

Arvidsson, L. 1982. A monograph of the lichen genus Coccocarpia. Opera Botanic 67: 1-96.

Awasthi, D.D. 1988. A key to the macrolichens of India and Nepal. Journal Hattori Botanical Laboratory 65: 207-302.

Blanco, O.; Crespo, A. \& Divakar, P.K. 2005. Molecular phylogeny of parmotremoid lichens (Ascomycota, Parmeliaceae). Mycologia 97(1): 150-159.

Brodo, I.M. 1973. Substrate ecology. Pp. 401-436. In: Hale, M.E. (Ed.). The Lichens. New York, Academic Press.

Brunialti, G. \& Giordani, P. 2003. Variability of lichen diversity in a climatically heterogeneous area (Liguria, NW Italy). Lichenologist 35: 55-69.

Dodge, C.W. 1964. Some lichens of tropical África. Nova Hedwigia: 179-184.

Eliasaro, S. 1992. Liquens do Gênero Heterodermia (PYXINACEAE, ASCOMYCOTINA) no Rio Grande do Sul, Brasil. Dissertação (Mestrado). Porto Alegre, Universidade Federal do Rio Grande do Sul.

Eliasaro, S. \& Adler, M. 2000. The species of Canomaculina, Myelochroa, Parmelinella and Parmelinopsis (PARMELIACEAE, Lichenized Ascomycotina) from the "Segundo Planalto" in the state of Paraná, Brazil. Acta Botanica Brasilica 14: 127-39.

Elix, J.A. 1994. Flora of Australia. Lichens - Lecanorales 2, Parmeliaceae. Australian Biological Resources Study 55: 5-360.

Elix, J.A.; Johnston, J. \& Verdon, D. 1986. Canoparmelia, Paraparmelia and Relicinopsis. Three new genera in the Parmeliaceae (Lichenized Ascomycotina). Mycotaxon 27: 271-282.

Elix, J.A. \& Hale, M.E. 1987. Canomaculina, Myelochroa, Parmelinella, Parmelinopsis and Parmotremopsis, five new genera in the Parmeliaceae (Lichenized, Ascomycotina). Mycotaxon 29: 233-244.

Filgueiras, T.S.; P.E. Nogueira; A.L. Brochado \& G.F. Guala II. 1994. Caminhamento: um método expedito para levantamentos florísticos qualitativos. Cadernos de Geociências 12: 39-43.

Fleig, M. 1985. Estudo Preliminar da Família PARMELIACEAE (Liquens) no Rio Grande do Sul, Brasil. Comunicação do Museu de Ciências PUCRS, Sér. Botânica 35: 79-91.

Fleig, M. 1990. Liquens da Estação Ecológica de Aracuri. Novas ocorrências. Iheringia 4: 121-125. 
Fleig, M. 1997. Os gêneros Parmotrema, Rimelia e Rimeliella (Lichenes - Ascomycotina, Parmeliaceae) no Rio Grande do Sul, Brasil. Tese (Doutorado). São Paulo, Universidade de São Paulo.

Fleig, M. 1999. O gênero Pseudocyphellaria (Liquens) no Rio Grande do Sul, Brasil. Pesquisas Botânica 49: 163-179.

Fleig, M.; Ahti, T. \& Stenroos, S. 1995. A família Cladoniaceae (Liquens) no Rio Grande do Sul. Napaea 11: 1-29.

Fleig, M. \& Grüninger, W. 2000. Levantamento preliminar dos liquens do Centro de Pesquisas e Conservação da natureza Pró-Mata, São Francisco de Paula, Rio Grande do Sul, Brasil. Napae 12: 5-20.

Fleig, M. \& Grüninger, W. 2008. Liquens da Floresta com Araucária no Rio Grande do Sul. Pró-Mata: Guia de Campo 3. Tübingen, University of Tübingen.

Galloway, D.J. 1985a. Nomenclatural notes on Pseudochyphellaria II: some southern hemisphere taxa. Lichenologist 17: 303-307.

Galloway, D.J. 1985b. Flora of New Zealand - Lichens. Wellington, P.D. Hasselberg.

Galloway, D.J. 1986a. Non-glabrous species of Pseudocyphellaria from southern South America. Lichenologist 18: 105-168.

Galloway, D.J. 1986b. Nomenclatural notes on Pseudochyphellaria III: some New Zealand taxa. Lichenologist 18: 289-291.

Galloway, D.J. 1988. Studies in Pseudocyphellaria (lichens) I. The New Zealand species. Bulletin of the British Museum (Natural History) 17: 1-267.

Galloway, D.J. 1994. Studies on the lichen genus Sticta (Schreber) Ach. I: Southern south American species. Lichenologist 26: 223-282.

Galloway, D.J. 1997. Studies on the lichen genus Sticta (Schreber) Ach.: IV: The New Zealand species. Lichenologist 29: 105-168.

Galloway, D.J. 1998a. Studies on the lichen genus Sticta (Schreber) Ach. V: Australian species. Tropical Bryologist 15: 117-160.

Galloway, D.J. 1998b. Edward Vainio and the family Lobariaceae, with special reference to the taxonomic history of Sticta. Pp 61-84. In: Marcelli, M.P. \& Ahti, T. (Eds.). Recollecting Edward August Vainio. São Paulo, CETESB.

Galloway, D.J. 2001. Lobariaceae. Pp. 37-101. In: Flora of Australia. Lichens 3. Australian Biological Resoucers Study 58A.

Galloway, D.J. \& James, P.W. 1980. Nomenclatural notes on Pseudocyphellaria in New Zealand. Lichenologist 12: 291-303.

Galloway, D.J. \& James, P.W. 1986. Species of Pseudocyphellaria Vainio (Lichenes), recorder in Delise's "Histoire des Lichens: Genre Sticta". Nova Hedwigia 42: 423-490.

Global Biodiversity Information Facility. 2009. In: http://data.gbif.org/ home.htm. (Acesso em 21/10/2009).

Hale, M.E., 1976a. A monograph of the lichen genus Pseudoparmelia Lynge (Parmeliaceae). Smithsonian Contributions to Botany 31: 1-62.

Hale, M.E. 1976 b. A monograph of the lichen genus Parmelina Hale (Parmeliaceae). Smithsonian Contributions to Botany 33: 1-69.

Hawksworth, D.L. 1975. Lichens - New Introductory, matter and supplementary. Index by Smith, A. L. 1921. Cambridge, The Richmond Publishing Co.

Holtan-Hartwig, J. 1993. The lichen genus Peltigera, exclusive of the $P$. canina group, in Norway. Sommerfeltia 15: 1-77.

IBGE, 2008. Mapas de Biomas e de Vegetação - Dados 2004 - IBGE - Estudo Brasileiro de Geografia e Estatística. In: www.ibge.gov.br. (Acesso em 4/9/2008).

Jørgensen, P.M. 1978. The lichen family Pannariaceae in Europe. Opera botanica 45: 1-123.

Jørgensen, P.M. \& James, P.W. 1983. Studies on some Leptogium species of western Europe. Lichenologist 15: 109-125.

Jørgensen, P.M. \& James, P.W. 2001. A new Erioderma taxon from the Azores. Lichenologist 33: 483-485.

Jüriado, I.; Paal, J. \& Liira, J. 2003. Epiphytic and epixylic lichen species diversity in Estonian natural forest. Biodiversity and Conservation 12: $1587-1607$

Käffer, M.I. \& Martins-Mazzitelli, S.M.A. 2005. Fungos liquenizados corticícolas e terrícolas da área da sub-bacia do Sinos e Taquari-Antas, Rio Grande do Sul, Brasil. Acta Botanica Brasilica 19(4): 815-819.

Kashiwadani, H. \& Kalb, K. 1993. The genus Ramalina in Brazil. Lichenologist 25(1): 1-31.
Krog, H. 1982. Punctelia, a new lichen genus in the Parmeliaceae. Nordic Journal of Botany 2(3): 287-292.

Lesica, P.; McCune, B. \& Cooper, S.V. 1991. Differences in lichen and bryophyte communities between old-growth and managed second-growth forest in the Swan Valley, Montana. Canadian Journal Botany 69: 1745- 1755.

Louwhoff, S.H.J.J. \& Elix, J.A. 1999. Parmotrema and allied lichen genera in Papua New Guinea. Bibliotheca Lichenologia 73: 1-152.

Marcelli, M.P. 1987. Ecologia dos liquens da região sul-sudeste, com especial atenção ao Itanhaém (SP). Tese (Doutorado). São Paulo, Universidade de São Paulo.

Marcelli, M.P. 1996. Biodiversity assessment in lichenized fungi: the necessary naive roll makers. Pp. 326. In: de Bicudo, C.E. \& Menezes, N.A. (Eds.). São Paulo, A first approach.

Marcelli, M.P. 1998. History of Brazilian Lichenology. Pp. 25-47. In: Marcelli, M.P. \& Seaward, M.R.D. (Eds.). Lichenology in Latin America: history, current knowledge and applications. São Paulo, CETESB.

Marcelli, M.P., Pereira, E.C. \& Iacomini, M. 1998. A bibliography on brazilian lichenology. Pp. 47-63. In: Marcelli, M.P. \& Seaward, M.R.D. (Eds.). Lichenology in Latin America: history, current knowledge and applications. São Paulo, CETESB.

Martins, S.M.A. 2006. Estudo da comunidade liquenizada epifítica em Dodonea viscosa L. na restinga do Parque Estadual de Itapuã, Viamão, RS. Tese de Doutorado. São Paulo, Instituto de Botânica da Secretaria de Estado do Meio Ambiente.

Martins-Mazzitelli, S.M.A.; Käffer, M.I. \& Cardoso, N. 1999. Liquens corticícolas de Porto Alegre, Rio Grande do Sul, Brasil. Iheringia Ser. Bot. 52: 55-63.

McDonald, T., Miadlikowska, J. \& Lutzoni, F. 2003. The lichen genus Sticta in the Great Smoky Moutains: a phylogenetic study of morphological, chemical and molecular data. The Bryologist 106: 61-79.

Ministério do Meio Ambiente. 2002. Projeto de conservação e utilização sustentável da diversidade biológica brasileira: relatório de atividades. Brasília, Biodiversidade - Brasil.

Moberg, R. 1990. The lichen genus Physcia in Central and South America. Nordic Journal of Botany 10: 319-342.

Nash III, T. H (Ed.). 2008. Lichen Biology. Cambridge, Cambridge University Press.

Osorio, H.S. 1982. Contribution to the lichen flora of Brazil IX. Lichens from the municipality of Torres, Rio Grande do Sul State. Mycotaxon 1(9): 347-350.

Osorio, H.S. \& Fleig, M. 1984. Contribution to the lichen flora of Brazi XV. Lichens from Torre Sul and Morro Itapeva, Torres, Rio Grande do Sul State. Comunicaciones Botanicas del Museo de Historia Natural de Montevideo 67(4): 1-7.

Osorio, H.S. \& Fleig, M. 1986a. Contribution to the lichen flora of Brazil XVII. Lichens from São Francisco de Paula, Rio Grande do Sul State. Comunicaciones Botanicas del Museo de Historia Natural de Montevideo 74(4): 1-4.

Osorio, H.S. \& Fleig, M. 1986b. Contribution to the lichen flora of Brazil XVIII. Lichens from Itaimbezinho, Rio Grande do Sul State. Comunicaciones Botanicas del Museo de Historia Natural de Montevideo 75(4): 1-8.

Osorio, H.S. \& Fleig, M. 1990. Contribution to the lichen flora of Brazil XXVII. Additions and corrections to the Rio Grande do Sul lichen flora. Comunicaciones Botanicas del Museo de Historia Natural de Montevideo 94(5): 1-6.

Ribeiro, C.H. 1998. A Família Parmeliaceae (Ascomycota liquenizados) em regiões montanhosas dos estados de Minas Gerais, Rio de Janeiro e São Paulo. Dissertação (Mestrado). São Paulo, Universidade de São Paulo.

Schmidt J.; Kricke, R. \& Feige, G.B. 2001. A measurements of bark pH with a modified flathead electrode. Lichenologist 33: 456-60.

Scutari, N.C. 1992. Estudios sobre PYXINACEAE foliosas (LECANORALES, ASCOMYCOTINA) de la Argentina, IV: chaves de los generos y las especies de la Provincia de Buenos Aires. Boletín Sociedad argentina botanica 28: 169-173.

Scutari, N.C. 1995a. Los Macroliquenes de Buenos Aires, I: Dirinaria, Heterodermia e Hyperphyscia (PHYSCIACEAE, ASCOMYCOTINA). Darwiniana 33: 149-176. 
Scutari, N.C. 1995b. Los Macrolíquenes de Buenos Aires, II: Phaeophyscia, Physcia y Pyxine (PHYSCIACEAE, ASCOMYCOTINA). Darwiniana 33: 211-231.

Sérusiaux, E. 1983. New data on the lichen genus Punctelia (PARMELIACEAE). Nordic Journal Botany 3: 517-520.

Sierk, H.A. 1964. The genus Leptogium in north America and north of Mexico. Bryologist 67: 1-317.

Sipman, H. 2002. Mason Hale's key to Parmotrema, revised edition: key to wide-lobed parmelioid species occurring in Tropical America (genera Canomaculina, Parmotrema, Rimelia, Rimeliella). In: www. bgbm.org/sipman/keys/Neioparmo.htm. (Acesso em 15/4/2002).

Sipman, H. \& Harris, R.C.1989. Lichens. Pp. 88-135. In: Tropical rain forest ecosystems. Liegth, H. \& Werger, M.J.A. (Eds.). Amsterdam, Elsevier Science Publishers.

Spielmann, A.A. 2006. Checklist of lichens and lichenicolous fungi of Rio Grande do Sul (Brazil). Caderno de Pesquisa, Sér. Bio. 18(2): 1-165.

Swinscow, T.D.V. \& Krog, H. 1998. Macrolichens of East Africa. British Museum (Natural History) London.

Tehler, A. 1996. Systematics, phylogeny and classification. Pp. 217-239. In: Nash III, T.H. (Ed.). Lichen Biology. Melbourne, Cambridge University Press.
Thomson Jr., J.W. 1950. The species of Peltigera of North America North of Mexico. The American Midland Naturalist 44(1): 1-68.

Thor, G. 1991. The Placement of Chiodecton sanguineum (syn. Chiodecton rubrocinctum) and Cryptothecia striata sp. nov. The Bryologist 94(3): 278-83.

Verdon, D., Filson, R.B. \& Henssen, A. 1992. Collemataceae. Pp. 15-192. In: Flora of Australia. Lichens - Introduction, Lecanorales 1. Australian Biological Resources Study 54.

Xavier Filho, L., Cavalcante, W.de A., Cavalcante, A.A.S. 1983. Estudio del Género Coenogonium em Brasil. Boletin da Sociedade Broteriana, Sér.2. 56: 115-121.

Will-Wolf, S.; Esseen, P.A. \& Neitlich, P. 2002. Monitoring biodiversity and ecosystem function: Forests. Pp. 203-222. In: Nimis, P.L.; Scheidegger, C. \& Wolseley, P.A. (Eds). Monitoring with lichens - Monitoring lichens.

Woseley, P.A. 1991. Observations on the composition and distribution of the 'Lobarion' in forests of south east Ásia. Pp. 217-243. In: Galloway, D.J. (Ed.). Tropical Lichens: Their systematics, conservation and ecology. Oxford, Clarendon Press.

Yoshimura, I. \& Osorio, H. 1975. Notes on Lobaria cuprea with purple pigment. Journal Hattori Botanical Laboratory 39: 263-268. 